Article

\title{
Controllability for Fuzzy Fractional Evolution Equations in Credibility Space
}

\author{
Azmat Ullah Khan Niazi ${ }^{1,2}\left(\mathbb{D}\right.$, Naveed Iqbal ${ }^{3}\left(\mathbb{D}\right.$, Rasool Shah ${ }^{4}\left(\mathbb{D}\right.$, Fongchan Wannalookkhee ${ }^{5}(\mathbb{C}$ \\ and Kamsing Nonlaopon $5, *$ (D) \\ 1 Faculty of Mathematics and Computational Science, Xiangtan University, Xiangtan 411105, China; \\ azmatniazi35@gmail.com \\ 2 Department of Mathematics and Statistics, University of Lahore, Sargodha 40100, Pakistan \\ 3 Department of Mathematics, College of Science, University of Ha'il, Ha'il 81481, Saudi Arabia; \\ naveediqbal1989@yahoo.com \\ 4 Department of Mathematics, Abdul Wali Khan University, Mardan 23200, Pakistan; \\ rasoolshahawkum@gmail.com \\ 5 Department of Mathematics, Faculty of Science, Khon Kaen University, Khon Kaen 40002, Thailand; \\ fongchan_wanna@kkumail.com \\ * Correspondence: nkamsi@kku.ac.th; Tel.: +668-6642-1582
}

Citation: Niazi, A.U.K.; Iqbal, N.; Shah, R.; Wannalookkhee, F.;

Nonlaopon, K. Controllability for Fuzzy Fractional Evolution Equations in Credibility Space. Fractal Fract. 2021, 5, 112. https://doi.org/ $10.3390 /$ fractalfract5030112

Academic Editors: António M. Lopes, Liping Chen and Bruce Henry

Received: 4 July 2021

Accepted: 1 September 2021

Published: 8 September 2021

Publisher's Note: MDPI stays neutral with regard to jurisdictional claims in published maps and institutional affiliations.

Copyright: (C) 2021 by the authors Licensee MDPI, Basel, Switzerland. This article is an open access article distributed under the terms and conditions of the Creative Commons Attribution (CC BY) license (https:/ / creativecommons.org/licenses/by/ $4.0 /)$.

\begin{abstract}
This article addresses exact controllability for Caputo fuzzy fractional evolution equations in the credibility space from the perspective of the Liu process. The class or problems considered here are Caputo fuzzy differential equations with Caputo derivatives of order $\beta \in(1,2),{ }_{0}^{C} D_{t}^{\beta} u(t, \zeta)=$ $A u(t, \zeta)+f(t, u(t, \zeta)) d C_{t}+B x(t) C x(t) d t$ with initial conditions $u(0)=u_{0}, u^{\prime}(0)=u_{1}$, where $u(t, \zeta)$ takes values from $U\left(\subset E_{N}\right), V\left(\subset E_{N}\right)$ is the other bounded space, and $E_{N}$ represents the set of all upper semi-continuously convex fuzzy numbers on $\mathbb{R}$. In addition, several numerical solutions have been provided to verify the correctness and effectiveness of the main result. Finally, an example is given, which expresses the fuzzy fractional differential equations.
\end{abstract}

Keywords: Liu process; Caputo fuzzy fractional differential equations; fuzzy process; credibility space

MSC: 26A33; 34K37

\section{Introduction}

In real-world phenomena, a large number of physical processes can be modeled using dynamical equations containing fractional-order derivatives [1]. The theory of fuzzy sets is continuously drawing the attention of researchers because of its rich applicability in several fields, including mechanics, electrical, engineering, processing signals, thermal systems, robotics and control, and many other fields [2,3]. Therefore, it has been an object of increasing interest for researchers during the past few years.

Until 2010, the concept in terms of Hukuhara differentiability [4] was unable to produce the vast and varied behavior of the crisp solution. However, later in 2012, a Riemann-Liouville H-derivative based on strongly generalized Hukuhara differentiability $[5,6]$ was defined by Allahviranloo and Salahshour [7,8]. They also defined a fuzzy Riemann-Liouville fractional derivative.

Differential equations with fractional derivatives are known as fractional differential equations. Owing to the study of fractional derivatives, it is clear that they arise universally for major mathematical reasons. There are various types of derivatives, such as Caputo and Riemann-Liouville $[9,10]$ derivatives.

Initially, Zadeh presented the concept of the fuzzy set in 1965 via the membership function. The most interesting field is that of fuzzy fractional differential equations. These are useful for analyzing phenomena where there is an inherent impression. Solutions of 
uniqueness and existence for fuzzy equations have been studied by Kwun et al. [11,12] and Lee et al. [13].

One of the most recent mathematical concepts is the theory of controlled processes in modern engineering to enable significant applications. Furthermore, due to various random factors that affect their behavior, actual systems under control do not allow for a strictly deterministic analysis. The random existence of a system's actions is taken into account in the theory of controlled processes.

Many scholars have worked on controlled processes. Concerning fuzzy systems, controllability in an $n$-dimensional fuzzy vector space for an impulsive semi-linear fuzzy differential equation (FDE) was proved by Kwun and Park [14]. Research on controllability with nonlocal conditions of semi-linear fuzzy integro-differential equations was performed by Park et al. [15]. The controllability of impulsive semi-linear fuzzy integro-differential equations was proved by Park et al. [16]. Research on the stability and controllability of fuzzy control set differential equations was performed by Phu and Dung [17]. Lee et al. [18] studied controllability with nonlocal initial conditions in a nonlinear fuzzy control system's $n$-dimensional fuzzy space $E_{N}^{n}$.

The controllability of a stochastic system of quasi-linear stochastic evolution equations in Hilbert space was studied by Balasubramanian [19] and Yuhu [20], who studied the controllability with time-variant coefficients of stochastic control systems. Arapostathis et al. investigated the controllability properties of stochastic differential systems characterized by linear controlled diffusion perturbed by bounded, smooth, uniformly Lipschitz nonlinearity [21]. Brownian-motion-driven stochastic differential equations are a mature branch of modern mathematics and have been studied for a long time. The Liu process [22] was used to drive a new form of FDE, which was described as follows:

$$
d X_{t}=f\left(X_{t}, t\right) d t+g\left(X_{t}, t\right) d C_{t}
$$

where $C_{t}$ is the standard Liu operation, while $f$ and $g$ are assigned functions. A fuzzy method is used to solve this type of equation. The solutions of uniqueness and existence of some special FDEs were discussed by Chen [23] for homogeneous FDEs. An approximate technique was studied by Liu [24] for solving uncertain differential equations. Young et al. [25] worked on exact controllability for abstract FDEs in credibility space by using the results of Liu [24]. In a credibility space, the exact controllability of abstract FDEs is expressed as follows:

$$
\begin{aligned}
d x(t, \theta) & =A x(t, \theta) d t+f(t, x(t, \theta)) d C_{t}+B u(t), t \in[0, T], \\
x(0) & =x_{0} . \\
{ }_{0}^{c} D_{t}^{\beta} u(t, \zeta) & =A u(t, \zeta)+f(t, u(t, \zeta)) d C_{t}+B x(t) C x(t) d t, \quad \beta \in(1,2), \\
u(0) & =u_{0}, \\
u^{\prime}(0) & =u_{1},
\end{aligned}
$$

where the state take values from two bounded spaces $U\left(\subset E_{N}\right)$ and $V\left(\subset E_{N}\right)$. The set of all upper semi-continuously convex fuzzy numbers on $\mathbb{R}$ is $E_{N}$ and the credibility space is $\left(\Theta, \mathcal{P}, C_{r}\right)$.

The state function $u:[0, T] \times\left(\Theta, \mathcal{P}, C_{r}\right) \rightarrow U$ is a fuzzy coefficient. $f:[0, T] \times U \rightarrow U$ is a fuzzy function, $x:[0, T] \times\left(\Theta, \mathcal{P}, C_{r}\right) \rightarrow V$ is a control function, $B$ and $C$ are $V$ to $U$ linear bounded operators. $u_{0} \in E_{N}$ is an initial value and $C_{t}$ is a standard Liu process.

The aim of this paper is to look into the existence of solutions to FDEs as well as their exact controllability. Some researchers have found results about fuzzy differential equations in the literature, but most of them were for first-order differential equations or fractional orders between $(0,1]$. In our work we have found results for Caputo derivatives of order $(1,2)$; see $[5,10,25]$ for more details. Our results are more complicated than the previous ones, and we require more boundary conditions than previous methods. Due to 
the change in boundary conditions, using Caputo derivatives, and for order $(1,2)$, almost all the results are original, but for previous results references have already been mentioned. The theory of fuzzy sets is continuously drawing the attention of researchers due to its rich suitability in various fields, including mechanics, engineering, electrical, thermal systems, robotics, control, and signal processing.

We go through some fundamental concepts relevant to Liu processes and fuzzy sets in Section 3. The existence of solutions to free FDEs is shown in Section 4. Finally, we show that the fuzzy differential equation is exactly controllable in Section 5.

\section{Preliminaries}

Let the family of all nonempty compact convex subsets of $\mathbb{R}$ be denoted by $M_{k}(\mathbb{R})$ and addition and scalar multiplication are also usually defined as $M_{k}(\mathbb{R})$. Let $A_{1}$ and $B_{1}$ be two nonempty bounded subsets of $\mathbb{R}$. The Hausdorff metric is used to define the distance between $A_{1}$ and $B_{1}$ as

$$
d\left(A_{1}, B_{1}\right)=\max \left\{\sup _{a_{1} \in A_{1}} \inf _{b_{1} \in B_{1}}\left\|a_{1}-b_{1}\right\|, \sup _{b_{1} \in B_{1}} \inf _{a_{1} \in A_{1}}\left\|a_{1}-b_{1}\right\|\right\},
$$

where $\|\cdot\|$ indicates the usual Euclidean norm in $\mathbb{R}$. Then it is clear that $\left(M_{k}(\mathbb{R}), d\right)$ becomes a separable and complete metric space [23]. Denote

$$
E^{n}=\{x: \mathbb{R} \rightarrow[0,1] \mid u \text { satisfies (i)-(iv) below }\},
$$

where

(i) $\quad x$ is normal, there exists an $x_{0} \in \mathbb{R}$ such that $x\left(x_{0}\right)=1$;

(ii) $\quad x$ is fuzzy convex, that is $x(\lambda t+(1-\lambda) s) \geq 2$;

(iii) $x$ is an upper semi-continuous function on $\mathbb{R}$ that is $x\left(t_{0}\right) \geq \lim _{k \rightarrow \infty} \overline{x\left(t_{k}\right)}$ for any $t_{k} \in \mathbb{R}(k=0,1,2, \ldots), t_{k} \rightarrow t_{0}$

(iv) $[x]^{0}=c l\{u \in \mathbb{R} \mid x(t)>0\}$ is compact.

For $1<\beta<2$, denote $[x]^{\beta}=\{t \in \mathbb{R} \mid u(t) \geq \beta\}$ and $[u]^{0}$ are nonempty compact convex sets in $\mathbb{R}[26]$. Then from (i)-(iv), it follows that $\beta$-level set $[x]^{\beta} t \in M_{k}(\mathbb{R})$ for all $1<\beta<2$. We can have scalar multiplication and addition in fuzzy number space $E^{n}$ by using Zadeh's extension principle as follows:

$$
[x \oplus y]^{\beta}=[x]^{\beta} \oplus[y]^{\beta}, \quad[k x]^{\beta}=k[y]^{\beta},
$$

where $x, y \in E^{n}, k \in \mathbb{R}$ and $1<\beta<2$.

Suppose that $E_{N}$ represents the set of all upper semi-continuously convex fuzzy numbers on $\mathbb{R}$.

Definition 1 ([27]). Define a complete metric $D_{L}$ by

$$
\begin{aligned}
D_{L}(x, y) & =\sup _{1<\beta<2} d_{L}\left\{[x]^{\beta},[y]^{\beta}\right\} \\
& =\sup _{1<\beta<2} \max \left\{\left|x_{l}^{\beta}-y_{l}^{\beta}\right|,\left|x_{l}^{\beta}-y_{r}^{\beta}\right|\right\},
\end{aligned}
$$

for any $u, v \in E_{N}$, which satisfies $D_{L}(x+z, y+z)=D_{L}(x, y)$ for each $z \in E_{N}$ and $[x]^{\alpha}=\left[x_{l}^{\beta}, u_{r}^{\beta}\right]$, for each $\beta \in(x, y)$ where $x_{l}^{\beta}, u_{r}^{\beta} \in \mathbb{R}$ with $x_{l}^{\beta} \leq u_{r}^{\beta}$.

Definition 2 ([28]). The Riemann-Liouville fractional derivative is defined as

$$
{ }_{a} D_{t}^{p} f(t)=\left(\frac{d}{d t}\right)^{n+1} \int_{a}^{t}(t-\tau)^{n-p} f(\tau) d \tau, \quad(n \leq p \leq n+1) .
$$


Definition 3 ([28]). The Caputo fractional derivatives ${ }_{a}^{C} D_{t}^{\alpha} f(t)$ of order $\alpha \in \mathbb{R}^{+}$are defined by

$$
{ }_{a}^{C} D_{t}^{\alpha} f(t)={ }_{a} D_{t}^{\alpha}\left(f(t)-\sum_{k=0}^{n-1} \frac{f^{(k)}(a)}{k !}(t-a)^{k}\right),
$$

where $n=[\alpha]+1$ for $\alpha \notin N_{0} ; n=\alpha$ for $\alpha \in N_{0}$.

In this paper, we consider a Caputo fractional derivative of order $1<\alpha \leq 2$, e.g.,

$$
{ }_{a}^{C} D_{t}^{3 / 2} f(t)={ }_{a} D_{t}^{3 / 2}\left(f(t)-\sum_{k=0}^{n-1} \frac{f^{(k)}(a)}{k !}(t-a)^{k}\right) .
$$

Definition 4 ([29]). The Wright function $\psi_{\alpha}$ is defined by

$$
\begin{aligned}
\psi_{\alpha}(\theta) & =\sum_{n=0}^{\infty} \frac{(-\theta)^{n}}{n ! \Gamma(-\alpha n+1-\alpha)} \\
& =\frac{1}{\pi} \sum_{n=1}^{\infty} \frac{(-\theta)^{n}}{(n-1) !} \Gamma(n \alpha) \sin (n \pi \alpha),
\end{aligned}
$$

where $\theta \in \mathbb{C}$ with $0<\alpha<1$.

Definition 5 ([30]). For any $x, y \in C\left([0, T], E_{N}\right)$, metric $H_{1}(x, y)$ on $C\left([0, T], E_{N}\right)$ is defined by

$$
H_{1}(x, y)=\sup _{0 \leq t \leq T} D_{L}(x(t), y(t)) .
$$

Allow $\Theta$ to be a nonempty set and $\mathcal{P}$ to be $\Theta$ 's power set. Each element of $\mathcal{P}$ is referred to as a case. To offer an axiomatic concept of credibility based on the assumption that $A$ will happen; to ensure that a number $C_{r}\left\{A_{1}\right\}$ is assigned to each event $A_{1}$, indicating the credibility of $A_{1}$ occurring; and to ensure the number $C_{r}\left\{A_{1}\right\}$ has certain mathematical properties that we intuitively predict, we accept the following four axioms:

(i) (Normality) $C_{r}\{\Theta\}=2$;

(ii) (Monotonicity) $C_{r}\left\{A_{1}\right\} \leq C_{r}\left\{B_{1}\right\}$, whenever $A_{1} \subset B_{1}$;

(iii) (Self-Duality) $C_{r}\left\{A_{1}\right\}+C_{r}\left\{A_{1}^{c}\right\}=2$ for any event $A_{1}$;

(iv) (Maximality) $C_{r}\left\{\cup_{i} A_{i}\right\}=\sup _{i} C_{r}\left\{A_{1}\right\}$ for any events $\left\{A_{i}\right\}$ with $\sup _{i} C_{r}\left\{A_{i}\right\}<1.5$.

Definition 6 ([31]). Let $\Theta$ be a nonempty set, $\mathcal{P}$ be $\Theta$ 's power set, and $C_{r}$ be a credibility measure. The triplet $\left(\Theta, \mathcal{P}, C_{r}\right)$ is then added to a set of real numbers.

Definition 7 ([31]). A fuzzy variable is a function from the set of real numbers $\left(\Theta, \mathcal{P}, C_{r}\right)$ to credibility space $\left(\Theta, \mathcal{P}, C_{r}\right)$.

Definition 8 ([31]). Let $\left(\Theta, \mathcal{P}, C_{r}\right)$ be a credibility space and $\left(\Theta, \mathcal{P}, C_{r}\right)$ be an index set. A fuzzy process is a function from a set of real numbers to $T \times\left(\Theta, \mathcal{P}, C_{r}\right)$.

That is, it is fuzzy process. $u(t, \zeta)$ is a two-variable function, with $u\left(t, \zeta^{*}\right)$ acting as a fuzzy variable for each $t^{*}$. The function $u(t, \zeta)$ is called the sample path of a fuzzy process for each fixed $\zeta^{*}$. If sampling is continuous for almost all $\zeta$, fuzzy process $u(t, \zeta)$ is said to be sample-continuous. We often use the symbol $u_{t}$ instead of $u(t, \zeta)$.

Definition 9 ([31]). A credibility space is known as $\left(\Theta, \mathcal{P}, C_{r}\right)$. For each $\beta \in(1,2)$, the $\beta$-level set is used for the fuzzy random variable $u_{t}$ in credibility space.

$$
\left[u_{t}\right]^{\beta}=\left[\left(u_{t}\right)_{l}^{\beta},\left(u_{t}\right)_{r}^{\beta}\right]
$$


is defined by

$$
\begin{aligned}
& \left(u_{t}\right)_{l}^{\beta}=\inf \left(u_{t}\right)^{\beta}=\inf \left\{a \in \mathbb{R} ; u_{t}(a) \geq \beta\right\}, \\
& \left(u_{t}\right)_{r}^{\beta}=\sup \left(u_{t}\right)^{\beta}=\inf \left\{a \in \mathbb{R} ; u_{t}(a) \geq \beta\right\},
\end{aligned}
$$

where $\left(u_{t}\right)_{l}^{\beta},\left(u_{t}\right)_{r}^{\beta} \in \mathbb{R}$ with $\left(u_{t}\right)_{l}^{\beta} \leq\left(u_{t}\right)_{r}^{\beta}$ when $\beta \in(1,2)$.

Definition 10 ([32]). Assume that $\theta$ is a fuzzy variable and that $r$ is a real number. Then $\theta^{\prime}$ s expected value is defined as

$$
E \theta=\int_{0}^{+\infty} C_{r}\{\theta \geq r\} d r-\int_{-\infty}^{0} C_{r}\{\theta \leq r\} d r
$$

provided that at least one integral is finite.

Lemma 1 ([32]). Assume that $\theta$ is a fuzzy vector. Below are the properties of the expected value operator E:

(i) if $f \leq g, E[f(\theta)] \leq E[g(\theta)]$;

(ii) $E[-f(\theta)]=-E[f(\theta)]$;

(iii) if $f$ and $g$ are comonotonic, we have for any nonnegative real numbers $a_{1}$ and $b_{1}$

$$
E\left[a_{1} f(\theta)+b_{1} g(\theta)\right]=a_{1} E[f(\theta)]+b_{1} E[g(\theta)],
$$

where $f(\theta)$ and $g(\theta)$ are fuzzy variables.

Definition 11 ([32]). A fuzzy process $C_{t}$ is a Liu process if

(i) $C_{0}=0$;

(ii) the $C_{t}$ has independent and stationary increments;

(iii) any increment $C_{t+s}-C_{s}$ is a normally distributed fuzzy variable with expected value et and variance $\phi^{2} t^{2}$, with membership function

$$
\xi(u)=2\left(1+\exp \left(\frac{\pi|u-e t|}{\sqrt{6} \phi t}\right)\right)^{-1}, \quad u \in \mathbb{R} .
$$

The diffusion and drift coefficients are the parameters $\phi$ and e, respectively. The Liu process is said to be standard if $e=0$ and $\phi=1$.

Definition 12 ([33]). Suppose $C_{t}$ to be a standard Liu process and $u_{t}$ to be a fuzzy process. The mesh is written as $c=t_{0}<\cdots<t_{n}=d$ for any partition of the closed interval $[c, d]$ with $c=t_{0}<\cdots<t_{n}=d$,

$$
\Delta=\max _{1 \leq i \leq n}\left(t_{i}-t_{i-1}\right) .
$$

The fuzzy integral of $u_{t}$ with respect to $C_{t}$ is then determined.

$$
\int_{c}^{d} u_{t} d C_{t}=\lim _{\Delta \rightarrow 0} \sum_{i=1}^{n} \mu\left(t_{i-1}\right)\left(C_{t_{i}}-C_{t_{i-1}}\right)
$$

provided that a limit exists almost certainly and is a fuzzy variable.

Lemma 2 ([33]). Let $C_{t}$ be a standard Liu process. The direction $C_{t}$ is Lipschitz continuous for any given with $C_{r}\{\zeta\}>0$, which implies that the following inequality holds:

$$
\left|C_{t_{1}}-C_{t_{2}}\right|<K(\zeta)\left|t_{1}-t_{2}\right|,
$$


where $K(\zeta)$ is the Lipschitz constant of a Liu process, which is a fuzzy variable defined by

$$
K(\zeta)= \begin{cases}\sup _{0 \leq s \leq t} \frac{\left|C_{t}-C_{s}\right|}{t}-s, & C_{r}\{\zeta\}>1 \\ \infty, & \text { otherwise }\end{cases}
$$

and $E\left[K^{p}\right]<\infty$ for all $p>1$.

Lemma 3 ([33]). Suppose $h(t ; c)$ to be a continuously differentiable function and $C_{t}$ to be standard Liu process. $u_{t}=h\left(t ; C_{t}\right)$ is the function to define. In addition, there is the chain rule that follows:

$$
d u_{t}=\frac{\partial h\left(t ; C_{t}\right)}{\partial t} d t+\frac{\partial h\left(t ; C_{t}\right)}{\partial C} d C_{t} .
$$

Lemma 4 ([33]). If $f(t)$ is a continuous fuzzy process, the below fuzzy integral inequality holds:

$$
\left|\int_{c}^{d} f(t) d C_{t}\right| \leq K \int_{c}^{d}|f(t)| d t
$$

The expression $K=K(\zeta)$ is defined in Lemma 2.

Definition 13. The fractional integral for a function $f$ with lower limit $t_{0}$ and order $\gamma$ can be defined as

$$
I_{t_{0}^{+}}^{\gamma} f(t)=\frac{1}{\Gamma(\gamma)} \int_{t_{0}^{+}}^{t} \frac{f(s)}{(t-s)^{1-\gamma}} d s, \quad \gamma>0, t>t_{0},
$$

where the right-hand side of the equality is defined point-wise on $\mathbb{R}^{+}$.

Lemma 5 ([34]). Let $\gamma>0$. Then

$$
I_{t_{0}+}^{\gamma}{ }^{c} D_{t_{0}^{+}}^{\gamma} f(t)=f(t)+c_{0}+c_{1} t+c_{2} t^{2}+\cdots+c_{n-1} t^{n-1}
$$

for some $c_{i} \in \mathbb{R}, i=0,1,2, \ldots, n-1$, where $n=[\gamma]+1$.

Lemma 6 ([35]). Let $\{C(t)\}_{t \in \mathbb{R}}$ be a strongly continuous cosine family in $X$ satisfying $\|C(t)\|_{L_{b}(X)} \leq M e^{\omega|t|}, t \in \mathbb{R}$, and let $A$ be the infinitesimal generator of $\{C(t)\}_{t \in \mathbb{R}}$, then for $\operatorname{Re} \lambda>\omega, \lambda^{2} \in \rho(A)$

$$
\lambda R\left(\lambda^{2} ; A\right) x=\int_{0}^{\infty} e^{-\lambda t} C(t) x d t, \text { and } R\left(\lambda^{2} ; A\right) x=\int_{0}^{\infty} e^{-\lambda t} S(t) x d t
$$

for $x \in X$.

\section{Existence of Solutions for Fuzzy Fractional Evolution Equations}

By Definition 8 , we use symbol $u_{t}$ instead of longer notation $u(t, \zeta)$ in this section. The uniqueness and existence of solutions for fuzzy differential Equation (3) $(x \equiv 0)$ are examined.

$$
\left\{\begin{array}{l}
{ }_{0}^{C} D_{t}^{\beta} u_{t}=A u_{t}+f\left(t, u_{t}\right) d C_{t}, \beta \in(1,2), \\
u(0)=u_{0} \\
u^{\prime}(0)=u_{1} \in E_{N}
\end{array}\right.
$$

where $u_{t}$ is a state that takes values from $U\left(\subset E_{N}\right)$. The set of all upper semi-continuously convex fuzzy numbers on $\mathbb{R}$ is labeled $E_{N},\left(\Theta, \mathcal{P}, C_{r}\right)$ is a credibility space, $A$ is a fuzzy coefficient, state function $u:[0, T] \times\left(\Theta, \mathcal{P}, C_{r}\right) \rightarrow U$ is a fuzzy process, $f:[0, T] \times U \rightarrow U$ is a regular fuzzy function, $C_{t}$ is a standard Liu process, and the initial value is $u_{0} \in E_{N}$. 
Lemma 7. If $u_{t}$ is a solution of (2) for $u(0)=u_{0}$, then $u_{t}$ is given by

$$
u_{t}=C_{q}(t) u_{0}+K_{q}(t) u_{1}+\int_{0}^{t}(t-s)^{q-1} P_{q}(t-s)\left[f\left(s, u_{s}\right)\right] d C_{s}+\int_{0}^{t}(t-s)^{q-1} B x(s) C x(s) d s,
$$

where $B$ and $C$ are linear bounded operators and

$$
\begin{aligned}
C_{q}(t) & =\int_{0}^{\infty} M_{q} C\left(t^{q} \zeta\right) d \zeta \\
K_{q}(t) & =\int_{0}^{t} C_{q}(s) d s \\
P_{q}(t) & =\int_{0}^{\infty} q \zeta M_{q} C\left(t^{q} \zeta\right) d \zeta \\
M_{q}\left(\zeta^{-q}\right) & =\psi_{q}(\zeta) \frac{\zeta^{q+1}}{q}
\end{aligned}
$$

such that $C_{q}(t)$ and $K_{q}(t)$ are continuous with $S(0)=I$ and $K(0)=I,\left|C_{q}(t)\right| \leq c, c>1$ and $\left|K_{q}(t)\right| \leq c, c>1$ for all $t \in[0, T]$.

Proof. Let $\operatorname{Re} \lambda>0$ and $\mathcal{L}$ be the Laplace transform

$$
\mu(\lambda)=\mathcal{L}[u(t)](\lambda)=\int_{0}^{\infty} e^{-\lambda s} u(s) d s, v(\lambda)=\mathcal{L}[f(t)](\lambda)=\int_{0}^{\infty} e^{-\lambda s} f(s) d s .
$$

According to Lemma 5, the Laplace transform is now being applied to Equation $u(t)=u_{0}+u_{1} t+\frac{1}{\Gamma(\beta)} \int_{0}^{t}(t-s)^{\beta-1}[A u(s)+f(s, u(s, \zeta))] d C_{s}+\int_{0}^{t}(t-s)^{\beta-1} B x(s) C x(s) d s$.

By Lemma 6, it follows that for $t \in[0, \infty)$.

Taking the Laplace transform on both sides of the above equation, we have

$$
\begin{aligned}
\mathcal{L}\left\{u_{t}\right\}= & \mathcal{L}\left\{u_{0}+u_{1} t\right\}+\mathcal{L}\left\{\frac{1}{\Gamma(\beta)} \int_{0}^{t}(t-s)^{\beta-1}[A u(s)+f(s, u(s, \zeta))] d C_{s}\right\} \\
& +\mathcal{L}\left\{\int_{0}^{t}(t-s)^{\beta-1} B x(s) C x(s) d s\right\}, \\
\mu(\lambda)= & \frac{1}{\lambda} u_{0}+\frac{1}{\lambda^{2}} u_{1}+\frac{1}{\lambda^{\beta}} A \mu(\lambda)+\frac{1}{\lambda^{\beta}} v(\lambda), \\
\mu(\lambda)-\frac{1}{\lambda^{\beta}} A \mu(\lambda)= & \frac{1}{\lambda} u_{0}+\frac{1}{\lambda^{2}} u_{1}+\frac{1}{\lambda^{\beta}} v(\lambda), \\
\mu(\lambda)\left(1-\frac{1}{\lambda^{\beta}} A\right)= & \frac{1}{\lambda} u_{0}+\frac{1}{\lambda^{2}} u_{1}+\frac{1}{\lambda^{\beta}} v(\lambda), \\
\mu(\lambda)= & \lambda^{\beta-1}\left(\lambda^{\beta}-A\right)^{-1} u_{0}+\lambda^{\beta-2}\left(\lambda^{\beta}-A\right)^{-1} u_{1}+\left(\lambda^{\beta}-A\right)^{-1} v(\lambda) .
\end{aligned}
$$

As a result, $t \geq 0$,

$$
\mu(\lambda)=\lambda^{\frac{\beta}{2}-1} \int_{0}^{\infty} e^{-\lambda^{\frac{\beta}{2}} t} C(t) u_{0} d s+\lambda^{-1} \lambda^{\frac{\beta}{2}-1} \int_{0}^{\infty} e^{-\lambda^{\frac{\beta}{2}} t} C(t) u_{1} d s+\int_{0}^{\infty} e^{-\lambda^{\frac{\beta}{2}} t} S(t) v(\lambda) d C_{t} d s .
$$

Let

$$
\psi_{q}(\zeta)=\frac{q}{\zeta^{q+1}} M_{q}\left(\zeta^{-q}\right), \quad \zeta \in(0, \infty) .
$$

Its Laplace transform is as follows:

$$
\int_{0}^{\infty} e^{-\lambda \zeta} \psi_{q}(\zeta) d \zeta=e^{-\lambda^{q}}
$$


for $q \in\left(\frac{1}{2}, 1\right)$.

To begin, we will use (6),

$$
\begin{aligned}
\lambda^{q-1} \int_{0}^{\infty} e^{-\lambda^{q} t} C(t) u_{0} d t & =\int_{0}^{\infty} q(\lambda t)^{q-1} e^{-(\lambda t)^{q} C\left(t^{q}\right) u_{0} d t} \\
& =\int_{0}^{\infty}-\frac{1}{\lambda} \frac{d}{d t}\left(\int_{0}^{\infty} e^{-\lambda t \zeta} \psi_{q}(\psi) d \psi\right) C\left(t^{q}\right) u_{0} d t \\
& =\int_{0}^{\infty} \int_{0}^{\infty} \zeta \psi_{q}(\zeta) e^{-\lambda t \zeta} C\left(t^{q}\right) u_{0} d \zeta d t \\
& =\int_{0}^{\infty} \int_{0}^{\infty} \psi_{q}(\zeta) e^{-\lambda t} C\left(\frac{t^{q}}{\zeta^{q}}\right) u_{0} d t d \zeta \\
& =\int_{0}^{\infty} e^{-\lambda t}\left[\int_{0}^{\infty} \psi_{q}(\zeta) C\left(\frac{t^{q}}{\zeta^{q}}\right) u_{0} d \zeta\right] d t \\
& =\mathcal{L}\left[\int_{0}^{\infty} M_{q}(\zeta) C\left(t^{q} \zeta\right) u_{0} d \zeta\right](\lambda) \\
& =\mathcal{L}\left[C_{q}(t) u_{0}\right](\lambda)
\end{aligned}
$$

Furthermore, by applying the Laplace convolution theorem, we obtain $\mathcal{L}\left[g_{1}(t)\right](\lambda)=\lambda^{-1}$.

$$
\begin{aligned}
\lambda^{-1} \lambda^{q-1} \int_{0}^{\infty} e^{-\lambda^{q} t} C(t) u_{1} d t & =\mathcal{L}\left[C_{q}(t) u_{1}\right](\lambda) \lambda^{-1} \lambda^{q-1} \int_{0}^{\infty} e^{-\lambda^{q} t} C(t) u_{1} d t \\
& =\mathcal{L}\left[g_{1} * C_{q}(t) u_{1}\right](\lambda) .
\end{aligned}
$$

Similarly, we observe

$$
\begin{aligned}
\int_{0}^{\infty} e^{-\lambda^{q} t} S(t) v(\lambda) d t & =\int_{0}^{\infty} q t^{q-1} e^{-(\lambda t)^{q}} S\left(t^{q}\right) v(\lambda) d t \\
= & \int_{0}^{\infty} \int_{0}^{\infty} q t^{q-1} \psi_{q}(\zeta) e^{-\lambda t \zeta} S\left(t^{q}\right) v(\lambda) d \zeta d t \\
& =\int_{0}^{\infty} \int_{0}^{\infty} q \frac{t^{q-1}}{\zeta^{q}} \psi_{q}(\zeta) e^{-\lambda t} S\left(\frac{t^{q}}{\zeta^{q}}\right) v(\lambda) d t d \zeta \\
& =\int_{0}^{\infty} e^{-\lambda t}\left[\int_{0}^{\infty} q \frac{t^{q-1}}{\zeta^{q}} \psi_{q}(\zeta) e^{-\lambda t}\left(\frac{t^{q}}{\zeta^{q}}\right) v(\lambda) d \zeta\right] d t \\
& =\mathcal{L}\left[\int_{0}^{\infty} q t^{q-1} M_{q}(\zeta) S\left(t^{q} \zeta\right) d \zeta\right](\lambda) \cdot \mathcal{L}[f(t)](\lambda) \\
& =\mathcal{L}\left[\int_{0}^{t}(t-s)^{q-1} P_{q}(t-s) f(s) d C_{s}+\int_{0}^{t}(t-s)^{q-1} P_{q}(t-s) B x(s) C x(s) d s\right](\lambda)
\end{aligned}
$$

Using the Laplace transform's uniqueness theorem and combining (7)-(9), we have the following

$$
\begin{aligned}
u_{t}= & \int_{0}^{\infty} \int_{0}^{\infty} \zeta \psi_{q}(\zeta) e^{-\lambda t \zeta} C\left(t^{q}\right) u_{0} d \zeta d t+\mathcal{L}\left[C_{q}(t) u_{1}\right](\lambda) \\
& +\lambda^{-1} \lambda^{q-1} \int_{0}^{\infty} e^{-\lambda{ }^{q} t} C(t) u_{1} d t+\int_{0}^{\infty} \int_{0}^{\infty} q t^{q-1} \psi_{q}(\zeta) e^{-\lambda t \zeta} S\left(t^{q}\right) v(\lambda) d \zeta d t . \\
u_{t}= & C_{q}(t) u_{0}+K_{q}(t) u_{1}+\int_{0}^{t}(t-s)^{q-1} P_{q}(t-s)\left[f\left(s, u_{s}\right)\right] d C_{s} \\
& +\int_{0}^{t}(t-s)^{q-1} P_{q}(t-s) B x(s) C x(s) d s .
\end{aligned}
$$

Assume that the following statements are true:

$\left(H_{1}\right)$ For $u_{t}, v_{t} \in C\left([0, T] \times\left(\Theta, \mathcal{P}, C_{r}\right), U\right), t \in[0, T]$. There exists a positive number $m$, such that

$$
d_{L}\left(\left[f\left(t, u_{t}\right)\right]^{\beta},\left[f\left(t, v_{t}\right)\right]^{\beta}\right) \leq m d_{L}\left(\left[u_{t}\right]^{\beta},\left[v_{t}\right]^{\beta}\right)
$$


and

$$
f\left(0, \mathcal{X}_{\{0\}}(0)\right) \equiv 0 \text {. }
$$

$\left(H_{2}\right) \quad 2 c m K T \leq 2$.

We know that (2) has solution $u_{t}$ because of Lemma 7. Thus, in Theorem 1 we show that the solution to (2) is unique.

Theorem 1. If $u_{0} \in E_{N}$, if $\left(H_{1}\right)$ and $\left(H_{2}\right)$ hold, (2) has a unique solution $u_{t} \in C([0, T]) \times$ $\left.\left(\Theta, \mathcal{P}, C_{r}\right), U\right)$.

Proof. For all $\theta_{t} \in C\left([0, T] \times\left(\Theta, \mathcal{P}, C_{r}\right), U\right), t \in[0, T]$, define

$$
\begin{aligned}
\phi \theta_{t}= & C_{q}(t) u_{0}+K_{q}(t) u_{1}+\int_{0}^{t}(t-s)^{q-1} P_{q}(t-s)\left[f\left(s, \theta_{s}\right)\right] d C_{s} \\
& +\int_{0}^{t}(t-s)^{q-1} P_{q}(t-s) B x(s) C x(s) d s .
\end{aligned}
$$

As a result, one can illustrate that $\phi \theta:[0, T] \times\left(\Theta, \mathcal{P}, C_{r}\right) \rightarrow C\left([0, T] \times\left(\Theta, \mathcal{P}, C_{r}\right), U\right)$ is continuous,

$$
\phi: C\left([0, T] \times\left(\Theta, \mathcal{P}, C_{r}\right), U\right) \rightarrow C\left([0, T] \times\left(\Theta, \mathcal{P}, C_{r}\right), U\right) .
$$

A fixed point of $\phi$ is also an obvious solution for Equation (2). By Lemma 4 and hypothesis $\left(H_{1}\right), \theta_{t}, \mu_{t} \in C\left([0, T] \times\left(\Theta, \mathcal{P}, C_{r}\right), U\right)$.

$$
\begin{aligned}
d_{L}\left(\left[\phi \theta_{t}\right]^{\beta},\left[\phi \mu_{t}\right]^{\beta}\right)= & d_{L}\left(\left[\int_{0}^{t}(t-s)^{q-1} P_{q}(t-s)\left[f\left(s, \theta_{s}\right)\right] d C_{s}+\int_{0}^{t}(t-s)^{q-1} P_{q}(t-s) B x(s) C x(s)\right]^{\beta},\right. \\
& {\left.\left[\int_{0}^{t}(t-s)^{q-1} P_{q}(t-s)\left[f\left(s, \mu_{s}\right)\right] d C_{s}+\int_{0}^{t}(t-s)^{q-1} P_{q}(t-s) B x(s) C x(s)\right]^{\beta}\right) } \\
\leq & 2 c m K \int_{0}^{t} d_{L}\left(\left[\theta_{s}\right]^{\beta},\left[\mu_{s}\right]^{\beta}\right) d s .
\end{aligned}
$$

Therefore, we obtain

$$
\begin{aligned}
D_{L}\left(\phi \theta_{t}, \phi \mu_{t}\right) & =\sup _{\beta \in(1,2)} d_{L}\left(\left[\phi \theta_{t}\right]^{\beta},\left[\phi \mu_{t}\right]^{\beta}\right) \\
& \leq 2 c m K \int_{0}^{t} \sup _{\beta \in(1,2)} d_{L}\left(\left[\theta_{t}\right]^{\beta},\left[\mu_{t}\right]^{\beta}\right) d s \\
& =2 c m K \int_{0}^{t} D_{L}\left(\theta_{s}, \mu_{s}\right) d s .
\end{aligned}
$$

As a consequence, by Lemma 1, for a.s. $\theta \in \Theta$,

$$
\begin{aligned}
E\left(H_{1}(\phi \theta, \phi \mu)\right) & =E\left(\sup _{t \in(0, T]} D_{L}\left(\phi \theta_{t}, \phi \mu_{t}\right)\right) \\
& \leq E\left(2 c m K \sup _{t \in(0, T]} \int_{0}^{t} D_{L}\left(\theta_{t}, \mu_{t}\right)\right) \\
& \leq 2 \operatorname{cmKTE}\left(H_{1}(\theta, \mu)\right) .
\end{aligned}
$$

By hypothesis $\left(H_{2}\right)$, a contraction mapping is $\phi$. This has a unique fixed point $x_{t} \in$ $C\left([0, T] \times\left(\Theta, \mathcal{P}, C_{r}\right), U\right)$ by the Banach fixed point theorem in Equation (2). 


\section{Exact Controllability for Fuzzy Fractional Evolution Equations}

The exact controllability of Caputo fuzzy differential Equation (3) is examined in this section. For each $x$ in $V\left(\subset E_{N}\right)$, we consider a solution for (3).

$$
\left\{\begin{aligned}
u_{t}= & C_{q}(t) u_{0}+K_{q}(t) u_{1}+\int_{0}^{t}(t-s)^{q-1} P_{q}(t-s) f\left(s, u_{s}\right) d C_{s} \\
& +\int_{0}^{t}(t-s)^{q-1} P_{q}(t-s) B x_{s} C x_{s} d s \\
u(0)= & u_{0} \\
u^{\prime}(0)= & u_{1}
\end{aligned}\right.
$$

where $S(t)$ is continuous with $S(0)=I$ and $S^{\prime}(0)=I,|S(t)| \leq c, c>0, t \in[0, T]$. For Caputo fuzzy differential equations, we define the concept of controllability.

Definition 14. Equation (3) is said to be controllable on $[0, T]$ if there is a control $u_{t} \in V$ for every $u_{0} \in E_{N}$ such that the solution $u$ of (3) satisfies $u_{t}=u^{-1} \in U$, a.s. $\zeta$ that is $\left[u_{t}\right]^{\beta}=\left[u^{1}\right]^{\beta}$.

Define fuzzy mapping $\tilde{G}: \tilde{P}(R) \rightarrow U$

$$
\tilde{G}^{\beta}(y)= \begin{cases}\int_{0}^{T}(t-s)^{q-1} P_{q}^{\beta}(t-s) B y_{s} C y_{s} d s, & y \subset \bar{\Gamma}_{x,} \\ 0, & \text { otherwise, }\end{cases}
$$

where $\bar{\Gamma}_{x}$ is the closure of support $x$ and $\tilde{P}(R)$ is a nonempty fuzzy subset of $\mathbb{R}$.

Then there is a $\tilde{G}_{i}^{\beta}(i=m, n)$,

$$
\begin{gathered}
\tilde{G}_{m}^{\beta}\left(y_{m}\right)=\int_{0}^{T}(t-s)^{q-1} P_{m}^{\beta}(t-s) B\left(y_{s}\right)_{m} C\left(y_{s}\right)_{m} d s,\left(y_{s}\right)_{m} \in\left[\left(y_{s}\right)_{m}^{\beta},\left(y_{s}\right)^{1}\right], \\
\tilde{G}_{n}^{\beta}\left(y_{n}\right)=\int_{0}^{T}(t-s)^{q-1} P_{n}^{\beta}(t-s) B\left(y_{s}\right)_{n} C\left(y_{s}\right)_{n} d s,\left(y_{s}\right)_{n} \in\left[\left(y_{s}\right)^{1},\left(y_{s}\right)_{n}^{\beta}\right] .
\end{gathered}
$$

We assume that $\tilde{G}_{m}^{\beta}, \tilde{G}_{n}^{\beta}$ are bijective mappings. A $\beta$-level set of $x_{s}$ can be represented as follows:

$$
\begin{aligned}
{\left[x_{s}\right]^{\beta}=} & {\left[\left(x_{s}\right)_{m}^{\beta},\left(x_{s}\right)_{n}^{\beta}\right] } \\
= & {\left[( \tilde { G } _ { m } ^ { \beta } ) ^ { - 1 } \left\{\left(u^{1}\right)_{m}^{\beta}-C_{q}(t)\left(u_{0}\right)_{m}^{\beta}-K_{q}(t)\left(u_{1}\right)-\int_{0}^{t}(t-s)^{q-1} P_{q}(t-s) f_{m}^{\beta}\left(s, u_{s}\right) d C_{s}\right.\right.} \\
& \left.-\int_{0}^{t}(t-s)^{q-1} P_{q}(t-s) B_{m}^{\beta}\left(x_{s}\right) C_{m}^{\beta}\left(x_{s}\right) d s\right\},\left(\tilde{G}_{n}^{\beta}\right)^{-1}\left\{\left(u^{1}\right)_{n}^{\beta}-C_{q}(t)\left(u_{0}\right)_{n}^{\beta}-K_{q}(t)\left(u_{1}\right)\right. \\
& \left.\left.-\int_{0}^{t}(t-s)^{q-1} P_{q}(t-s) f_{n}^{\beta}\left(s, u_{s}\right) d C_{s}-\int_{0}^{t}(t-s)^{q-1} P_{q}(t-s) B_{n}^{\beta}\left(x_{s}\right) C_{n}^{\beta}\left(x_{s}\right) d s\right\}\right] .
\end{aligned}
$$

The $\beta$-level of $x_{t}$ is obtained by substituting this expression into (10). 


$$
\begin{aligned}
{\left[u_{t}\right]^{\beta}=} & {\left[C_{q}(t) u_{0}+K_{q}(t) u_{1}+\int_{0}^{t}(t-s)^{q-1} P_{q}(t-s) f\left(s, u_{s}\right) d C_{s}\right.} \\
& \left.+\int_{0}^{t}(t-s)^{q-1} P_{q}(t-s) B x_{s} C x_{s} d s\right]^{\beta} \\
= & {\left[C_{m}^{\beta}(t)\left(u_{0}\right)_{m}^{\beta}+K_{m}^{\beta}(t)\left(u_{1}\right)_{m}^{\beta}+\int_{0}^{t}(t-s)^{q-1} P_{m}^{\beta}(t-s) f\left(s,\left(u_{s}\right)_{m}^{\beta}\right) d C_{s}\right.} \\
& +\int_{0}^{t}(t-s)^{q-1} P_{m}^{\beta}(t-s) B\left(\tilde{G}_{m}^{\beta}\right)^{-1}\left\{\left(u^{1}\right)_{m}^{\beta}-C_{q}(t)\left(u_{0}\right)_{m}^{\beta}-K_{q}(t)\left(u_{1}\right)_{m}^{\beta}\right. \\
& \left.-\int_{0}^{t}(t-s)^{q-1} P_{q}(t-s) f_{m}^{\beta}\left(s, u_{s}\right) d C_{s}-\int_{0}^{t}(t-s)^{q-1} P_{q}(t-s) B_{m}^{\beta}\left(x_{s}\right) C_{m}^{\beta}\left(x_{s}\right) d s\right\} d s, \\
& C_{n}^{\beta}(t)\left(u_{0}\right)_{n}^{\beta}+K_{n}^{\beta}(t)\left(u_{1}\right)_{n}^{\beta}+\int_{0}^{t}(t-s)^{q-1} P_{n}^{\beta}(t-s) f\left(s,\left(u_{s}\right)_{n}^{\beta}\right) d C_{s} \\
& +\int_{0}^{t}(t-s)^{q-1} P_{n}^{\beta}(t-s) B\left(\tilde{G}_{n}^{\beta}\right)^{-1}\left\{\left(u^{1}\right)_{n}^{\beta}-C_{q}(t)\left(u_{0}\right)_{n}^{\beta}-K_{q}(t)\left(u_{1}\right)_{n}^{\beta}\right. \\
& \left.\left.-\int_{0}^{t}(t-s)^{q-1} P_{q}(t-s) f_{n}^{\beta}\left(s, u_{s}\right) d C_{s}-\int_{0}^{t}(t-s)^{q-1} P_{q}(t-s) B_{n}^{\beta}\left(x_{s}\right) C_{n}^{\beta}\left(x_{s}\right) d s\right\} d s\right] \\
= & {\left[C_{m}^{\beta}(t)\left(u_{0}\right)_{m}^{\beta}+K_{m}^{\beta}(t)\left(u_{1}\right)_{m}^{\beta}+\int_{0}^{t}(t-s)^{q-1} P_{m}^{\beta}(t-s) f\left(s,\left(u_{s}\right)_{m}^{\beta}\right) d C_{s}\right.} \\
& +\tilde{G}_{m}^{\beta}\left(\tilde{G}_{m}^{\beta}\right)^{-1}\left\{\left(u^{1}\right)_{m}^{\beta}-C_{q}(t)\left(u_{0}\right)_{m}^{\beta}-K_{q}(t)\left(u_{1}\right)_{m}^{\beta}-\int_{0}^{t}(t-s)^{q-1} P_{q}(t-s) f_{m}^{\beta}\left(s, u_{s}\right) d C_{s}\right. \\
& \left.\left.-\int_{0}^{t}(t-s)^{q-1} P_{q}(t-s) B_{m}^{\beta}\left(x_{s}\right) C_{m}^{\beta}\left(x_{s}\right) d s\right\} d s, C_{n}^{\beta} t\right)\left(u_{0}\right)_{n}^{\beta}+K_{n}^{\beta}(t)\left(u_{1}\right)_{n}^{\beta} \\
& +\int_{0}^{t}(t-s)^{q-1} P_{n}^{\beta}(t-s) f\left(s,\left(u_{s}\right)_{n}^{\beta}\right) d C_{s}+\tilde{G}_{n}^{\beta}\left(\tilde{G}_{n}^{\beta}\right)^{-1}\left\{\left(u^{1}\right)_{n}^{\beta}-C_{q}(t)\left(u_{0}\right)_{n}^{\beta}-K_{q}(t)\left(u_{1}\right)_{n}^{\beta}\right. \\
= & {\left[\left(u^{1}\right)_{m}^{\beta},\left(u^{1}\right)_{n}^{\beta}\right] } \\
= & {\left[u^{1}\right]^{\alpha} . }
\end{aligned}
$$

Hence this control $x_{t}$ satisfies $u_{t}=u^{1}$, a.s. $\zeta$.

We now set

$$
\begin{aligned}
\psi u_{t}= & C_{q}(t) u_{0}+K_{q}(t) u_{1}+\int_{0}^{t}(t-s)^{q-1} P_{q}(t-s) f\left(s, u_{s}\right) d C_{s} \\
& +\int_{0}^{t}(t-s)^{q-1} P_{q}(t-s) B \tilde{G}^{-1}\left\{u^{1}-C_{q}(t) u_{0}-K_{q}(t) u_{1}\right. \\
& -\int_{0}^{t}(t-s)^{q-1} P_{q}(t-s) f\left(s, u_{s}\right) d C_{t} \\
& \left.-\int_{0}^{t}(t-s)^{q-1} P_{q}(t-s) B\left(x_{s}\right) C\left(x_{s}\right) d s\right\} d s .
\end{aligned}
$$

Fuzzy mapping $\tilde{G}^{-1}$ satisfies the above statement.

Theorem 2. If Lemma 4 and the hypotheses $\left(H_{1}\right)$ and $\left(H_{2}\right)$ are satisfied, then Equation (3) is controllable on $[0, T]$.

Proof. We can easily verify that $\psi$ is continuous from $C([0, T] \times(\Theta, \mathcal{P}, U)$ to $C([0, T]$. For any given $\zeta$ with $C_{r}\{\zeta\}>0, x_{t}, y_{t} \in C\left([0, T] \times\left(\Theta, \mathcal{P}, C_{r}\right), U\right)$, we have by Lemma 4 and hypotheses $\left(H_{1}\right)$ and $\left(H_{2}\right)$ that 


$$
\begin{aligned}
\left.d_{L}\left(\left[\psi u_{t}\right]^{\beta},\left[\psi v_{t}\right]^{\beta}\right]\right)= & d_{L}\left(\left[C_{q}(t) u_{0}+K_{q}(t) u_{1}+\int_{0}^{t}(t-s)^{q-1} P_{q}(t-s) f\left(s, u_{s}\right) d C_{s}+\int_{0}^{t}(t-s)^{q-1} P_{q}(t-s)\right.\right. \\
& B \tilde{G}^{-1}\left\{u^{1}-C_{q}(t) u_{0}-K_{q}(t) u_{1}-\int_{0}^{t}(t-s)^{q-1} P_{q}(t-s) f\left(s, u_{s}\right) d C_{t}\right. \\
& \left.-\int_{0}^{t}(t-s)^{q-1} P_{q}(t-s) B\left(x_{s}\right) C\left(x_{s}\right) d s\right\} d s, C_{q}(t) v_{0}+K_{q}(t) v_{1}+\int_{0}^{t}(t-s)^{q-1} P_{q}(t-s) \\
& f\left(s, v_{s}\right) d C_{s}+\int_{0}^{t}(t)^{q-1} P_{q}(t-s) B \tilde{G}^{-1}\left\{v^{1}-C_{q}(t) v_{0}-K_{q}(t) v_{1}\right. \\
& \left.\left.-\int_{0}^{t}(t-s)^{q-1} P_{q}(t-s) f\left(s, v_{s}\right) d C_{t}-\int_{0}^{t}(t-s)^{q-1} P_{q}(t-s) B\left(x_{s}\right) C\left(x_{s}\right) d s\right\} d s\right] \\
\leq & d_{L}\left(\left[\int_{0}^{t}(t-s)^{q-1} P_{q}(t-s) f\left(s, u_{s}\right) d C_{s}\right]^{\beta},\left[\int_{0}^{t}(t-s)^{q-1} P_{q}(t-s) f\left(s, v_{s}\right) d C_{s}\right]^{\beta}\right) \\
& +d_{L}\left(\left[\int_{0}^{t}(t-s)^{q-1} P_{q}(t-s) B \tilde{G}^{-1} \times \int_{0}^{t}(t-s)^{q-1} P_{q}(t-s) f\left(s, u_{t}\right) d C_{t}(s) d s\right]^{\beta},\right. \\
& {\left[\int_{0}^{t}(t-s)^{q-1} P_{q}(t-s) B \tilde{G}^{-1} \times \int_{0}^{t}(t-s)^{q-1} P_{q}(t-s) f\left(s, v_{t} d C_{t}(s) d s\right]^{\beta}\right) } \\
\leq & c m K \int_{0}^{t} d_{L}\left(\left[u_{s}\right]^{\beta},\left[v_{s}\right]^{\beta}\right) d s+d_{L}\left(\left[\tilde{G} \tilde{G}^{-1} \int_{0}^{t}(t-s)^{q-1} P_{q}(t-s) f\left(s, u_{t}\right) d C_{t}(s)\right]^{\beta},\right. \\
& {\left.\left[\tilde{G} \tilde{G}^{-1} \int_{0}^{t}(t-s)^{q-1} P_{q}(t-s) f\left(s, v_{t}\right) d C_{t}(s)\right]^{\beta}\right) } \\
\leq & c m K \int_{0}^{t} d_{L}\left(\left[u_{s}\right]^{\beta},\left[v_{s}\right]^{\beta}\right) d s+c m K \int_{0}^{t} d_{L}\left(\left[f\left(s, u_{s}\right)\right]^{\beta},\left[f\left(s, u_{s}\right)\right]^{\beta}\right) d s \\
\leq & 2 c m K \int_{0}^{t} d_{L}\left(\left[u_{s}\right]^{\beta},\left[v_{s}\right]^{\beta}\right) d s .
\end{aligned}
$$

Therefore, by Lemma 1,

$$
\begin{aligned}
E\left(H_{1}(\psi u, \psi v)\right) & =E\left(\sup _{t \in[0, T]} D_{L}\left(\psi u_{t}, \psi v_{t}\right)\right) \\
& =E\left(\sup _{t \in[0, T]} \sup _{1<\beta \leq 2} D_{L}\left(\left|\psi u_{t}\right|^{\beta},\left|\psi v_{t}\right|^{\beta}\right) d s\right) \\
& \leq E\left(\sup _{t \in[0, T]} \sup _{1<\beta \leq 2} 2 c m K \int_{0}^{t} D_{L}\left(\left[u_{s}\right]^{\beta},\left[v_{s}\right]^{\beta}\right) d s\right) \\
& \leq E\left(\sup _{t \in[0, T]} 2 c m K \int_{0}^{t} D_{L}\left(u_{s}, v_{s}\right) d s\right) \\
& \leq 2 c m K T F\left(H_{1}(u, v)\right) .
\end{aligned}
$$

Thus, $(2 c m K T)<2$ is a sufficiently small $T$. As a consequence, $\psi$ represents a contraction mapping. Banach fixed point theorem is now used to prove that Equation (10) has a unique fixed point. As a consequence, (3) can be controlled on $[0, T]$.

Example 1. In credibility space, we consider the following Caputo fuzzy fractional differential equations

$$
\left\{\begin{array}{l}
D^{\beta} u(t, \zeta)=A u(t, \zeta) d t+f(t, x(t, \zeta)) d c_{t}+B x(t) C x(t) d t \\
u(0)=u_{0} \\
u^{\prime}(0)=u_{1} \in E_{N}
\end{array}\right.
$$


where the state takes values from two bounded spaces $U\left(\subset E_{N}\right)$ and $V\left(\subset E_{N}\right)$. The set of all upper semi-continuously convex fuzzy numbers on $R$ is $E_{N}$ and the credibility space is $\left(\Theta, \mathcal{P}, C_{r}\right)$.

The state function $u:[0, T] \times\left(\Theta, \mathcal{P}, C_{r}\right) \rightarrow U$ is a fuzzy coefficient. $f:[0, T] \times U \rightarrow U$ is a fuzzy process. $x:[0, T] \times\left(\Theta, \mathcal{P}, C_{r}\right) \rightarrow V$ is a regular fuzzy function, $x:[0, T] \times\left(\Theta, \mathcal{P}, C_{r}\right) \rightarrow$ $V$ is a control function, and $B$ is $a V$ to $U$ linear bounded operator. $u_{0} \in E_{N}$ is an initial value and $C_{t}$ is a standard Liu process.

Suppose $f\left(t, u_{t}\right)=\tilde{2} t u_{t}, S^{-1}(t)=e^{-\tilde{2} t}$, defining $w_{t}=S^{-1}(t) u_{t}$. Then the equations of balance become

$$
\left\{\begin{aligned}
u_{t}= & C_{q}(t) u_{0}+K_{q}(t) u_{1}+\int_{0}^{t}(t-s)^{q-1} P_{q}(t-s) \tilde{2} t u_{t} d C_{s} \\
& +\int_{0}^{t}(t-s)^{q-1} P_{q}(t-s) B x_{s} C x_{s} d s \\
u(0)= & u_{0} \\
u^{\prime}(0)= & u_{1} \in E_{N} .
\end{aligned}\right.
$$

Therefore, Lemma 7 is satisfied.

Since $[2]^{\beta}=[\beta+1,3-\beta]$ is the $\beta$-level set of fuzzy number $\tilde{2}$ for all $\beta \in(1,2)$, the $\beta$-level set of $f\left(t, u_{t}\right)$ is

$$
\left[f\left(t, u_{t}\right)\right]^{\beta}=t\left[(\beta+1)\left(u_{t}\right)_{m}^{\beta},(3-\beta)\left(u_{t}\right)_{m}^{\beta}\right] .
$$

Further, we have

$$
\begin{aligned}
d_{L}\left(\left[f\left(t, u_{t}\right)\right]^{\beta},\left[f\left(t, v_{t}\right)\right]^{\beta}\right) & =d_{L}\left(t\left[(\beta+1)\left(u_{t}\right)_{m}^{\beta},(\beta+1)\left(u_{t}\right)_{n}^{\beta}\right], t\left[(\beta+1)\left(v_{t}\right)_{m}^{\beta},(\beta+1)\left(v_{t}\right)_{n}^{\beta}\right]\right) \\
& =t \max \left\{(\beta+1)\left|\left(u_{t}\right)_{m}^{\beta}-\left(v_{t}\right)_{m}^{\beta}\right|,(3-\beta)\left|\left(u_{t}\right)_{n}^{\beta}-\left(v_{t}\right)_{n}^{\beta}\right|\right\} \\
& \leq 3 T \max \left\{\left|\left(u_{t}\right)_{m}^{\beta}-\left(v_{t}\right)_{m}^{\beta}\right|,\left|\left(u_{t}\right)_{n}^{\beta}-\left(v_{t}\right)_{n}^{\beta}\right|\right\} \\
& =m d_{L}\left(\left[u_{t}\right]^{\beta},\left[v_{t}\right]^{\beta}\right),
\end{aligned}
$$

where $m=3 T$ satisfies an inequality in the $\left(H_{1}\right),\left(H_{2}\right)$ hypotheses. After that, all of the conditions defined in Theorem 1 are satisfied.

Let $\tilde{1}$ be an initial value for $u_{0} . u^{1}=\tilde{2}$ is the target set. The $\beta$-level set of fuzzy number $\tilde{1}$ is $[\tilde{1}]=[\beta-2,2-\beta], \beta \in(1,2)$. The $\beta$-level set of $x_{s}$ of $(10)$ is introduced.

$$
\begin{aligned}
{\left[x_{s}\right]=} & {\left[\left(x_{s}\right)_{m}^{\beta},\left(x_{s}\right)_{n}^{\beta}\right] } \\
= & {\left[\left(\tilde{G}_{m}^{\beta}\right)^{-1}\left\{(\beta+2)-S_{m}^{\beta}(\beta-2)-\int_{0}^{T} S_{m}^{\beta}(T-s) s(\beta+1)\left(u_{s}\right)_{m}^{\beta} d C_{s}\right\},\right.} \\
& \left.\left(\tilde{G}_{n}^{\beta}\right)^{-1}\left\{(3-\beta)-S_{n}^{\beta}(3-\beta)-\int_{0}^{T} S_{n}^{\beta}(T-s) s(3-\beta)\left(u_{s}\right)_{n}^{\beta} d C_{s}\right\}\right] .
\end{aligned}
$$

The $\beta$-level of $u_{t}$ is then obtained by substituting this expression into (12).

$$
\begin{aligned}
{\left[u_{t}\right]^{\beta}=} & {\left[S_{m}^{\beta}(T)(\beta-2)+\int_{0}^{T} S_{m}^{\beta}(T-s) s(\beta+2)\left(u_{s}\right)_{m}^{\beta} d C_{s}+\int_{0}^{T} S_{m}^{\beta}(T-s) B\left(\tilde{G}_{m}^{\beta}\right)^{-1}\right.} \\
& \left\{(\beta+2)-S_{m}^{\beta}(T)(\beta-2)-\int_{0}^{T} S_{m}^{\beta}(T-s) s(\beta+2)\left(u_{s}\right)_{m}^{\beta} d C_{s}\right\} d s, \\
& S_{n}^{\beta}(T)(2-\beta)+\int_{0}^{T} S_{n}^{\beta}(T-s) s(3-\beta)\left(u_{s}\right)_{n}^{\beta} d C_{s}+\int_{0}^{T} S_{n}^{\beta}(T-s) B\left(\tilde{G}_{n}^{\beta}\right)^{-1} \\
& \left.\left\{(3-\beta)-S_{r}^{\beta}(T)(2-\beta)-\int_{0}^{T} S_{n}^{\beta}(T-s) s(3-\beta)\left(u_{s}\right)_{n}^{\beta} d C_{s}\right\} d s\right] \\
= & {[(\beta+2),(3-\beta)] } \\
= & {[\tilde{2}]^{\beta} . }
\end{aligned}
$$

After that, all conditions described in Theorem 2 are satisfied. As a result, (13) can be controllable on $[0, T]$. 
Example 2. Assume the following fuzzy fractional evolution equation in credibility space

$$
{ }_{0}^{C} D_{t}^{1.5} u(t, \zeta)=A u(t, \zeta) d t+f\left(t^{3}+2 t^{2}+4 t\right) d c_{t}+5 B x(t) C x(t) d t,
$$

with initial conditions $u(0)=u_{0}, u^{\prime}(0)=u_{1} \in E_{N}, \beta \in 1.5$, where the state takes values from two bounded spaces $U\left(\subset E_{N}\right)$ and $V\left(\subset E_{N}\right)$. The set of all upper semi-continuously convex fuzzy numbers on $R$ is $E_{N}$ and the credibility space is $\left(\Theta, \mathcal{P}, C_{r}\right)$.

The state function $u:[0, T] \times\left(\Theta, \mathcal{P}, C_{r}\right) \rightarrow U$ is a fuzzy coefficient. $f:[0, T] \times U \rightarrow U$ is a fuzzy process. $x:[0, T] \times\left(\Theta, \mathcal{P}, C_{r}\right) \rightarrow V$ is a regular fuzzy function, $x:[0, T] \times\left(\Theta, \mathcal{P}, C_{r}\right) \rightarrow$ $V$ is a control function, and $B$ is a $V$ to $U$ linear bounded operator. $u_{0} \in E_{N}$ is an initial value and $C_{t}$ is a standard Liu process.

Since $[4]^{\beta}=[\beta+3,5-\beta]$ is the $\beta$-level set of fuzzy number $\tilde{2}$ for all $\beta \in(1,2)$, the $\beta$-level set of $f\left(t, u_{t}\right)$ is

$$
\left[f\left(t, u_{t}\right)\right]^{\beta}=t\left[(1.5+3)\left(u_{t}\right)_{m}^{1.5},(5-1.5)\left(u_{t}\right)_{m}^{1.5}\right] .
$$

Further, we have

$$
\begin{aligned}
d_{L}\left(\left[f\left(t, u_{t}\right)\right]^{1.5},\left[f\left(t, v_{t}\right)\right]^{1.5}\right) & =d_{L}\left(t\left[(1.5+3)\left(u_{t}\right)_{m}^{1.5},(1.5+3)\left(u_{t}\right)_{n}^{1.5}\right], t\left[(1.5+1)\left(v_{t}\right)_{m}^{1.5},(1.5+1)\left(v_{t}\right)_{n}^{1.5}\right]\right) \\
& =t \max \left\{(1.5+3)\left|\left(u_{t}\right)_{m}^{1.5}-\left(v_{t}\right)_{m}^{1.5}\right|,(5-1.5)\left|\left(u_{t}\right)_{n}^{1.5}-\left(v_{t}\right)_{n}^{1.5}\right|\right\} \\
& \leq 5 T \max \left\{\left|\left(u_{t}\right)_{m}^{1.5}-\left(v_{t}\right)_{m}^{1.5}\right|,\left|\left(u_{t}\right)_{n}^{1.5}-\left(v_{t}\right)_{n}^{1.5}\right|\right\} \\
& =m d_{L}\left(\left[u_{t}\right]^{1.5},\left[v_{t}\right]^{1.5}\right),
\end{aligned}
$$

where $m=5 T$ satisfies an inequality in the $\left(H_{1}\right),\left(H_{2}\right)$ hypotheses.

\section{Conclusions}

If exact controllability is encouraged for fuzzy fractional evolution equations, it can serve as a benchmark for treating controllability for equations in credibility space, such as fuzzy semi-linear integro-differential equations and fuzzy delay integro-differential equations. As a result, this study's theoretical result can be used to create stochastic extensions in credibility space. Moreover, future work may include expanding the ideas set out in this work, introducing observability, and generalizing other works. This is a fruitful field with wide research projects, which can lead to countless applications and theories. We plan to allocate notable attention to this direction.

Author Contributions: Conceptualization, A.U.K.N., N.I., and K.N.; investigation, N.I., R.S., F.W., and K.N.; methodology, A.U.K.N., N.I., R.S., F.W., and K.N.; validation, R.S., F.W., and K.N.; visualization, R.S., F.W., K.N.; writing-original draft, A.U.K.N., N.I., R.S., and K.N.; writing-review and editing, N.I., and K.N. All authors have read and agreed to the published version of the manuscript.

Funding: This research received no external funding.

Institutional Review Board Statement: Not applicable.

Informed Consent Statement: Not applicable.

Data Availability Statement: Not applicable.

Acknowledgments: The fourth author was supported by the Development and Promotion of Science and Technology Talents Project (DPST), Thailand.

Conflicts of Interest: The authors declare no conflicts of interest.

\section{References}

1. Miller, K.S.; Ross, B. An Introduction to the Fractional Calculus and Fractional Differential Equations; Wiley: Hoboken, NJ, USA, 1993.

2. Ahmad, B.; Ntouyas, S.K.; Agarwal, R.P.; Alsaedi, A. On fractional differential equations and inclusions with nonlocal and average-valued (integral) boundary conditions. Adv. Differ. Equ. 2016, 80. [CrossRef]

3. Mansouri, S.S.; Gachpazan, M.; Fard, O.S. Existence, uniqueness and stability of fuzzy fractional differential equations with local Lipschitz and linear growth conditions. Adv. Differ. Equ. 2017, 240. [CrossRef] 
4. Agarwal, R.P.; Lakshmikantham, V.; Nieto, J.J. On the concept of solution for fractional differential equations with uncertainty. Nonlinear Anal. Theory Methods Appl. 2010, 72, 2859-2862. [CrossRef]

5. Chakraverty, S.; Tapaswini, S.; Behera, D. Fuzzy Arbitrary Order System; John Wiley and Sons: Hoboken, NJ, USA, 2016.

6. Bede, B.; Stefanini, L. Generalized differentiability of fuzzy-valued functions. Fuzzy Sets Syst. 2013, 230, 119-141. [CrossRef]

7. Allahviranloo, T.; Salahshour, S.; Abbasbandy, S. Explicit solutions of fractional differential equations with uncertainty. Soft Comput. 2012, 16, 297-302. [CrossRef]

8. Salahshour, S.; Allahviranloo, T.; Abbasbandy, S. Solving fuzzy fractional differential equations by fuzzy Laplace transforms. Commun. Nonlinear Sci. Numer. Simul. 2012, 17, 1372-1381. [CrossRef]

9. Lakshmikantham, V.; Leela, S.; Devi, J.V. Theory of Fractional Dynamic Systems; Cambridge Academic Publishers: Cambridge, UK, 2009.

10. Lakshmikantham, V.; Vatsala, A.S. Basic theory of fractional differential equations. Nonlinear Anal. Theory Methods Appl. 2008, 69, 2677-2682. [CrossRef]

11. Kwun, Y.C.; Kim, W.H.; Nakagiri, S.I.; Park, J.H. Existence and uniqueness of solutions for the fuzzy differential equations in n-dimension fuzzy vector space. Int. J. Fuzzy Log. Intell. Syst. 2009, 9, 16-19. [CrossRef]

12. Kwun, Y.C.; Kim, J.S.; Hwang, J.S.; Park, J.H. Existence of solutions for the impulsive semilinear fuzzy intergrodifferential equations with nonlocal conditions and forcing term with memory in $n$-dimensional fuzzy vector space. Int. J. Fuzzy Log. Intell. Syst. 2011, 11, 25-32. [CrossRef]

13. Lee, B.Y.; Kwun, Y.C.; Ahn, Y.C.; Park, J.H. The existence and uniqueness of fuzzy solutions for semilinear fuzzy integrodifferential equations using integral contractor. Int. J. Fuzzy Log. Intell. Syst. 2009, 9, 339-342. [CrossRef]

14. Kwun, Y.C.; Park, M.J.; Kim, J.S.; Park, J.S.; Park, J.H. Controllability for the impulsive semilinear fuzzy differential equation in $n$-dimension fuzzy vector space. In Proceedings of the 2009 Sixth International Conference on Fuzzy Systems and Knowledge Discovery, Tianjin, China, 14-16 August 2009; pp. 45-48.

15. Park, J.H.; Park, J.S.; Kwun, Y.C. Controllability for the semilinear fuzzy integrodifferential equations with nonlocal conditions. In International Conference on Fuzzy Systems and Knowledge Discovery; Springer: Berlin/Heidelberg, Germany, 2006 ; pp. 221-230.

16. Park, J.H.; Park, J.S.; Ahn, Y.C.; Kwun, Y.C. Controllability for the impulsive semilinear fuzzy integrodifferential equations. In Fuzzy Information and Engineering; Springer: Berlin/Heidelberg, Germany, 2007; pp. 704-713.

17. Phu, N.D.; Dung, L.Q. On the stability and controllability of fuzzy control set differential equations. Int. J. Reliab. Saf. 2011, 5, 320-335. [CrossRef]

18. Lee, B.Y.; Park, D.G.; Choi, G.T.; Kwun, Y.C. Controllability for the nonlinear fuzzy control system with nonlocal initial condition in $E^{n}$ N. Int. J. Fuzzy Log. Intell. Syst. 2006, 6, 15-20. [CrossRef]

19. Balasubramaniam, P.; Dauer, J.P. Controllability of semilinear stochastic evolution equations in Hilbert space. J. Appl. Math. Stoch. Anal. 2001, 14, 329-339. [CrossRef]

20. Feng, Y. Convergence theorems for fuzzy random variables and fuzzy martingales. Fuzzy Sets Syst. 1999, 103, 435-441. [CrossRef]

21. Arapostathis, A.; George, R.K.; Ghosh, M.K. On the controllability of a class of nonlinear stochastic systems. Syst. Control Lett. 2001, 44, 25-34. [CrossRef]

22. Liu, B. Fuzzy process, hybrid process and uncertain process. J. Uncertain Syst. 2008, 2, 3-16.

23. Chen, X.; Qin, Z. A New Existence and Uniqueness Theorem for Fuzzy Differential Equations. Int. J. Fuzzy Syst. 2011, 13, 10.30000/IJFS.201106.0010.

24. Liu, Y. An analytic method for solving uncertain differential equations. J. Uncertain Syst. 2012, 6, $244-249$.

25. Lee, B.Y.; Youm, H.E.; Kim, J.S. Exact controllability for fuzzy differential equations in credibility space. Int. J. Fuzzy Log. Intell. Syst. 2014, 14, 145-153. [CrossRef]

26. Diamond, P.; Kloeden, P. Metric Spaces of Fuzzy Sets: Theory and Applications; World Scientific: Singapore, 1994.

27. Wang, G.; Li, Y.; Wen, C. On fuzzy n-cell numbers and n-dimension fuzzy vectors. Fuzzy Sets Syst. 2007, 158, 71-84. [CrossRef]

28. San, D. Podlubny, I.: Fractional Differential Equations; Academic Press: New York, NY, USA,1999.

29. Mainardi, F.; Paradisi, P.; Gorenflo, R. Probability distributions generated by fractional diffusion equations. arXiv 2007, arXiv:0704.0320.

30. Kwun, Y.; Kim, J.; Park, M.; Park, J. Nonlocal controllability for the semilinear fuzzy integrodifferential equations $n$-dimensional fuzzy vector space. Adv. Differ. Equ. 2009, 734090. [CrossRef]

31. Liu, B. A survey of credibility theory. Fuzzy Optim. Decis. Mak. 2006, 5, 387-408. [CrossRef]

32. Liu, B.; Liu, Y.K. Expected value of fuzzy variable and fuzzy expected value models. IEEE Trans Fuzzy Syst. 2002, 10, 445-450.

33. Fei, W. Uniqueness of solutions to fuzzy differential equations driven by Liu's process with non-Lipschitz coefficients. In Proceedings of the 2009 Sixth International Conference on Fuzzy Systems and Knowledge Discovery, Tianjin, China, 14-16 August 2009; pp. 565-569.

34. Zhang, S. Positive solutions for boundary-value problems of nonlinear fractional differential equations.Electron. J. Differ. Equ. 2006, 36, 1-12. [CrossRef]

35. Travis, C.C.; Webb, G.F. Cosine families and abstract nonlinear second order differential equations. Acta Math. Hung. 1978, 32, 75-96. [CrossRef] 